\title{
Origen y desarrollo del fenómeno mileurista en España: el caso de El País (2005-2011)
}

\author{
Cayetano FERnÁndeZ Romero \\ Universidad de San Jorge \\ cfernandez@usj.es \\ Alfonso CORRAL GARCÍA \\ Universidad de San Jorge \\ acorral@usj.es \\ Anabel ABAD Villamor \\ Universidad de San Jorge \\ aabad@alumni.usj.es
}

Recibido: 10/05/2012

Aceptado: 19/11/2012

\section{Resumen}

En el año 2005 se publicó por primera vez el término mileurista en el diario El País. Desde entonces, este neologismo se ha extendido y se ha generalizado entre la sociedad española. A través del siguiente trabajo seguimos el ciclo de vida de dicho término en este medio de comunicación y presentamos el perfil del mileurista, que se cotejará con la información procedente de instituciones oficiales con la finalidad de estudiar su evolución, tratamiento y representación. Del mismo modo nuestro objetivo consiste en establecer una serie de paradigmas que revelen las características de este fenómeno social made in Spain. Palabras clave: Mileurista, El País, juventud, clase social, generación.

\section{Origin and Development of the Mileurista Phenomenon in Spain: Case of El Pais (2005-2011)}

\begin{abstract}
In 2005 the term 'mileurista' (worker earning 1.000 euros a month) was published for the first time in the daily paper 'El País'. Since then, this new term has spread and become generally used within Spanish society. In the following study we follow the life cycle of this term in this means of communication and we present a profile of the 'mileurista', which will be compared with the information from official institutions with the aim of studying its evolution, treatment and representation. In the same way our objective consist of establishing a series of paradigms which reveale the characteristics of this social phenomenon which has been 'made in Spain'.
\end{abstract}

Keywords: Mileurista, El País, youth, social class, generation.

\section{Referencia normalizada}

FERNÁNDEZ ROMERO, Cayetano; CORRAL GARCÍA, Alfonso; y ABAD VILLAMOR, Anabel (2013): “Origen y desarrollo del fenómeno mileurista en España: el caso de El País (2005-2011)”. Estudios sobre el mensaje periodístico. Vol. 19, Núm. 1, págs.: 107-130. Madrid, Servicio de Publicaciones de la Universidad Complutense.

Sumario: 1. Introducción. El mileurista y la construcción social de la realidad. 2. Metodología y fuentes. 3. Resultados. 4. El perfil del mileurista; 4.1. Ser mileurista o aspirar a serlo; 4.2. Edad y género; 4.2.1. El género del mileurista; 4.3. Salario y situación laboral del mileurista; 4.4. Formación académica de los mileuristas; 4.5. Vivienda. ¿Con mis padres, solo o compartiendo?; 4.6. Mileurista y clase social: "Adiós, clase media, adiós". 5. Conclusiones. 6. Referencias bibliográficas. 


\section{Introducción. El mileurista y la construcción social de la realidad}

Cuando se lee el término mileurista se llega enseguida a la siguiente conclusión: persona que cobra mil euros. No es así. O por lo menos no solamente, pues abarca un concepto mucho más amplio y complejo. Una ciudadana desconocida, Carolina Alguacil, fue la primera que acuñó el término y lo hizo en la sección de El País por medio de una carta al director. En agosto de 2005 denunció su situación y la de tantos otros jóvenes españoles que del mismo modo recibieron y difundieron ese mensaje. "Pocas veces hemos asistido de una manera tan clara y tajante al nacimiento de un término que defendiera y describiera con tanto éxito una generación" (Freire, 2006: 11). ¿Cómo lo definió? "El mileurista es aquel joven, de 25 a 34 años, licenciado, bien preparado, que habla idiomas, tiene posgrados, másteres y cursillos".

Sin percatarse de las repercusiones de esa carta, Carolina Alguacil creó un nuevo topic para los medios de comunicación y la sociedad en general. Al hablar de un topic nos referimos al problema o cuestión clave a la que los políticos deberían estar prestando atención mientras no se le da la importancia percibida (Lang y Lang, 1981: 441). La realidad es que, por lo general, son los propios medios de comunicación, y en menor medida los políticos, quienes determinan los temas que forman parte del debate social, de la opinión pública. Es lo que se conoce como la teoría de la agendasetting (McCombs y Shaw, 1972).

Como señala Sádaba (2008: 80-81), esta teoría abarca tanto la fijación de las agendas, donde los medios tienen un papel especial para ofrecer los problemas de interés público, como su construcción por medio de distintas fuentes sociales. A esta segunda parte se le conoce como agenda building y está relacionada con la generación y creación de los contenidos de los medios. En este punto, una figura clave es la del gatekeeper, aquella persona que decide los temas sociales que penetran en los medios y cuáles no (López-Escobar y Sánchez de la Yncera, 1990). Además, con Cáceres (2011: 322 ), "es en las rutinas productivas del periodismo en donde se libran los ajustes necesarios para hacer compatibles afinidades e intereses diversos, económicos, comer-

\footnotetext{
1 "Normalmente iniciado en la hostelería, ha pasado grandes temporadas en trabajos no remunerados, llamados eufemísticamente becarios, prácticos (claro), trainings, etcétera. Ahora echa la vista atrás, y quiere sentirse satisfecho, porque al cabo de dos renovaciones de contrato, le han hecho fijo, en un trabajo que de alguna forma puede considerarse formal, 'lo que yo buscaba'. Lleva entonces tres o cuatro años en el circuito laboral, con suerte la mitad cotizados. Y puede considerarse ya un especialista, un ejecutivo; lo malo es que no gana más de mil euros, sin pagas extras, y mejor no te quejes. El mileurista hace tiempo que decidió irse de casa, y gasta más de un tercio de su sueldo en alquiler, porque le gusta disfrutar de la gran ciudad. Comparte piso con más gente, a veces es divertido, pero ya cansa. [...] El mileurista no ahorra, no tiene casa, no tiene coche, no tiene hijos, vive al día. A veces es divertido, pero ya cansa. El mileurista ha ido a 'Europa' este verano, en uno de esos vuelos baratos donde te hablan de tú, y ha dormido en un hostal joven (qué divertido). El mileurista ha pagado lo mismo por un café, incluso menos por la comida, que en su ciudad. Pregunta, investiga y allí los alquileres son parecidos, y piensa que España está ya al nivel europeo. Pero lo malo es que se ríen cuando dice que gana "nine hundred and ninety seven euros" (Alguacil, 2005).
} 
ciales, ideológicos, políticos o meramente coyunturales, más o menos reconocidos por los profesionales".

Eso sí, el paso del gatekeeping al newsmaking evidencia el sentido de ciertas lógicas sociales de producción cultural que estructuran la imagen de los acontecimientos mediáticos (Sierra Caballero, 1999: 326). El profesional de la comunicación tiene unos valores que determinan la selección de los hechos noticiosos: las características sustantivas del contenido, la disponibilidad del material y los criterios relativos al propio producto informativo, el público y la competencia periodística (Golding y Elliott, 1979). Estos cuatro principios determinan la actividad informativa de los emisores en función de la escasez de tiempo, la carencia de medios y la organización del trabajo (Sierra Caballero, 1999: 329). Al mismo tiempo, en este proceso entran en juego unos criterios que fijan la noticiabilidad de los hechos y que también son cumplidos en el caso del mileurismo: frecuencia, umbral, ausencia de ambigüedad, significatividad, consonancia, imprevisibilidad, continuidad, composición y valores socioculturales (Galtung y Ruge, 1980; Rodrigo, 1989: 111).

Por lo tanto, con esa carta al director Carolina Alguacil se adelantó a los periodistas. Este género periodístico cumple un papel trascendental en la prensa, pues su función principal radica en el intercambio con los lectores, principio vital para la supervivencia del periodismo. Hay autores que dudan sobre la legitimidad de las cartas al director como género periodístico de opinión. Sin embargo, el caso que estamos analizando demuestra su importancia en la práctica periodística en cuanto que generan y alimentan debates sociales (Cordova, 2011: 201). De este modo, rompiendo con los circuitos clásicos, el concepto de mileurismo surgió desde abajo, desde la crítica de una figura irrelevante que analizaba el estado socioeconómico de su generación.

Hasta la implantación y el auge de Internet, las personas individuales y, por tanto, la sociedad en general, solamente podían acercarse a los medios de comunicación a través de las cartas al director. Sin embargo, en la actualidad, gracias a Internet y las redes sociales, todas las personas pueden ejercer su periodismo, en tanto que consumen al tiempo que producen. Así, Ramonet (2011: 23-24) afirma que "estamos pasando de la era de los medios de masas a la de la masa de los medios". Se refiere al nuevo periodismo ciudadano, que tiene muchos tintes de periodismo social ${ }^{2}$ y que se ha visto tan desarrollado en 2011 de la mano de redes sociales como Twitter. Buena muestra de ello son las revoluciones árabes en un nivel internacional, o el movimiento del $15 \mathrm{M}$ a nivel nacional.

Entonces, ¿cómo es esta generación mileurista-periodística? En este estudio hemos tratado de ilustrarla a partir de las páginas de El País, con el objetivo de corroborar si los textos publicados se adecuan a la definición de Alguacil. Es conocido que un “joven, para serlo verdaderamente, habrá de situarse en relación con otros jóvenes, y

2 "El periodismo social es un periodismo que asume su papel como protagonista de los procesos sociales y reflexiona sobre su responsabilidad en los mismo. Su objetivo principal es que la comunicación sirva para generar un mejor diálogo entre los distintos actores de la sociedad. [...] Busca devolver una visión más amplia que ayude a la construcción de una sociedad más inclusiva" (Cytrynblum, 2009: 73). 
así encontrar su identidad específica dentro de la juventud. Especialmente habrá de situarse respecto a los jóvenes más cercanos a él o a ella, su grupo de interacción, los amigos y los conocidos, pero también respecto a las imágenes juveniles que recibe de los medios de comunicación social" (Revilla, 2011: 119).

Por lo tanto, a decir verdad, al mileurista se le conoce mejor por sus problemas que por sus virtudes ${ }^{3}$. Estos problemas son similares a los de sus predecesores y muchos de ellos los heredarán sus sucesores. "Hasta que se inventó el término, se hablaba de la otra generación de los 80, la que había nacido durante esos años, o de la generación X, o de los JASP (Jóvenes, aunque sobradamente preparados). También se habló de los GP (Guapos y pobres) o de la Generación del Milenio. Ninguna de esas etiquetas funcionó de verdad" (Freire, 2006: 16). Por el contrario, otra de las denominaciones que sí ha alcanzado cierto éxito es la de generación Peter Pan, la de unos jóvenes que han chocado con su realidad. Una juventud cuyo único refugio es la vuelta a la etapa juvenil recuperando sus mismas actitudes y ocios ${ }^{4}$.

Entre todas estas nomenclaturas se debe encuadrar al mileurista, ese desencantado, cínico, que conoce su derecho a protestar y lo ejerce, pero que en pocos casos da el paso siguiente: no denuncia, no exige, no pacta (Freire, 2006: 29). Sin embargo, el mileurista ha sabido crecer y evolucionar en su propio seno, llegando incluso a aplacar estas críticas. El mileurista ha pasado a formar parte de una nueva corriente que le ha sustituido por varias razones, entre las que destaca la reivindicación de una sociedad más justa, más ecuánime. Hablamos del indignado (Hessel, 2011), cuyo movimiento en España ha sido conocido como el 15M. Por todos estos motivos, el concepto parece hoy superado y, por ello, así se analizará desde el ciclo de vida del término y su escritura gráfica en El País.

Como los medios de comunicación tienen un estimable valor demoscópico, ya que no solo generan opinión, sino que también son receptores de las sensibilidades sociales (Motis et al., 2010), en este trabajo se estudiará si el perfil que se puede trazar a partir de las noticias publicadas en El País a lo largo de estos seis años corresponde con el de Alguacil o si, por el contrario, se percibe una evolución o transformación del mismo. Además, en la medida de lo posible, se cotejará con las características que definen a los mileuristas según los datos oficiales publicados por instituciones oficiales, como el Instituto Nacional de Estadística (INE), el Consejo de la Juventud de España (CJE), el Observatorio Joven de Empleo (OBJOVEM) y el Observatorio Joven de Vivienda (OBJOVI).

\footnotetext{
3 "Sueldos bajos, precios elevados de vivienda, falta de reconocimiento, dificultades de emancipación, diferencia entre expectativas creadas y la realidad, una educación que infravalorado la capacidad de frustración y ha potenciado el conocimiento académico, baja educación emocional, crisis de valores familiares y emocionales. A eso se le une una sociedad tecnológica y cambiante, que al ciudadano como un consumidor y valora sobre todas las cosas la sexualidad y la juventud" (Freire, 2006: 29).

4 Se trata de "una generación desencantada, desorientada, perpleja, aplastada, con sensación de pesadez, con enormes y constantes dudas porque el mapa de rutas que trazaron sus padres ya no les sirve y han de orientarse con uno nuevo en blanco y con unos valores diferentes" (Garriga, 2009: 32).
} 


\section{Metodología y fuentes}

Nuestro objeto de estudio se centra en el análisis de la evolución, el tratamiento y la representación que El País realiza sobre la figura del mileurista. Se ha elegido este diario porque en él se publicó por primera vez el término y, además, no hay que olvidar que se trata del periódico de información general más leído de España ${ }^{5}$. Por este motivo, se ha optado por llevar a cabo un análisis de contenido con la finalidad principal de conocer este fenómeno social desde el horizonte mediático. Esta técnica de investigación permite describir de forma objetiva, sistemática y cuantitativa el contenido manifiesto de la comunicación (Berelson, 1952) para conseguir inferencias reproducibles y válidas que pueden aplicarse a su contexto (Krippendorff, 1990: 28).

De este modo, a partir de sus vertientes cuantitativa y cualitativa se han analizado un total de 211 piezas periodísticas que corresponden al intervalo 21 de agosto de 2005, momento en que Alguacil definía con precisión el término mileurista, y el 20 de noviembre de 2011, cuando se celebraron las últimas elecciones generales que determinaron el triunfo del Partido Popular después de dos mandatos socialistas. Inicialmente el estudio se concentraba en el periodo correspondiente a la publicación de dos textos relacionados con Alguacil en los años 2005 (cuando redactó la carta al director) y 2010 (una entrevista con motivo del quinto aniversario de su publicación).

Sin embargo, el espacio se amplió hasta las elecciones del $20 \mathrm{~N}$ puesto que en mayo de 2010 surgió el denominado movimiento $15 \mathrm{M}$, también conocido como el de los indignados (Aunión, 2011: 26-27). En torno a este fenómeno podemos encontrar una gran variedad de personas según la edad, sexo, educación y poder adquisitivo. Entre todos estos perfiles también encontramos a los denominados mileuristas. Como los indignados tuvieron un especial protagonismo en los días previos y posteriores a las últimas elecciones municipales celebras en mayo de 2011, que se propagó hasta las generales de noviembre, nos pareció oportuno prolongar el periodo de estudio hasta el $20 \mathrm{~N}$ con el objetivo de poder detectar demandas y reivindicaciones de este movimiento que hicieran referencia al grupo de los mileuristas.

\section{Resultados}

Al comprobar la distribución de estas 211 publicaciones por secciones (figura 1), destacan las 104 registradas en los suplementos regionales de Cataluña, Madrid, Andalucía, Galicia, Comunidad Valenciana o País Vasco. Uno de los motivos que pueden explicar esta preocupación regional lo encontramos en el hecho de que el contenido de estas publicaciones facilita la identificación con la cotidianeidad de los habitantes en dichas Comunidades. Por otro lado, en segundo lugar aparece la sección de opinión (37), expresando así cierto nivel de crítica social.

${ }^{5}$ Los datos generales del Estudio General de Medios (EGM), que elabora la Asociación para la Investigación de los Medios de Comunicación (AIMC), para el último año móvil (tres últimas oleadas, octubre 2010 - mayo 2011) determinan un total de 1.961 .000 lectores. Por otro lado, es el diario nacional de información general con mayor difusión con 431.033 ejemplares (AEDE, 2010: 46-47). Su línea editorial, sus principios liberales y sociales quedan definidos en Libro de estilo de El País (El País, 2004). 


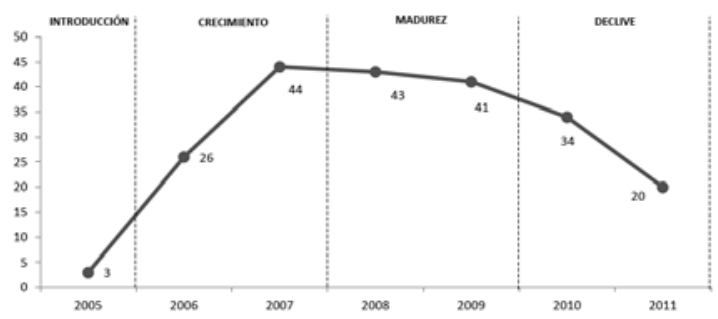

Figura 1. Ciclo de vida del término mileurista.

Fuente: Elaboración propia a partir de datos de El País

En esta sección el género más numeroso son las cartas al director, en las que aparecen varias declaraciones de personas mileuristas, testimonios que se han ido recogiendo a lo largo de este trabajo para aportar más detalles al perfil propuesto por $E l$ País. No en vano, "lo mejor de la función pública de la prensa lo encontramos en sus páginas de opinión. Significan un debate plural, un espacio donde se ejerce la libertad de expresión y donde se alcanzan espacios de conocimiento que la información no provee" (Casals, 2004: 61).

En cualquier caso, si bien las cartas al director son elegidas previamente a la publicación por un equipo de redactores de El País, no representan al periódico, por lo que hemos decidido incluir únicamente aquellos ejemplos de cartas al director que amplíen alguna característica al perfil del mileurista propuesto por el diario, dejando constancia de cuál es el perfil de El País y cuáles las aportaciones de sus lectores ${ }^{6}$.

Si analizamos la evolución anual del número de piezas aparecidas (figura 1) se observa cierto paralelismo con un desarrollado concepto del área del Marketing: el ciclo de vida del producto. Así, de acuerdo con Santesmases (2007: 456), este ciclo de vida es el proceso cronológico que transcurre desde el nacimiento o lanzamiento del producto en el mercado hasta su muerte o desaparición. Además, esta evolución cronológica presenta cuatro fases diferenciadas que también guardan estrecha relación con los resultados obtenidos: introducción (relativo a 2005), crecimiento (2006-2007), madurez (2008-2009) y declive (2010-2011).

Ahora, si se cambiase el término 'producto' por cualquier otra idea, concepto o palabra, tal y como puede ser mileurista, el axioma serviría por igual. Al mismo tiempo, el ciclo de vida se muestra condicionado por la alterabilidad y mutabilidad de los comportamientos, los hábitos, los entornos, las tendencias u otras alternativas (Santesmases, 2007: 455-457), que en el caso del mileurista podrían proceder de la de la aparición de otros conceptos que acumulan horizontes más amplios. Un ejemplo tácito se encuentra en los $\mathrm{JASP}^{7}$, término que surgió del mercado publicitario automo-

${ }^{6}$ Los ejemplos que proceden de cartas al director contienen en su cita la denominación "Carta al Director" inmediatamente después del nombre del periódico.

7 "Pía Codina, de 26 años, pertenece a un colectivo entre los inframileuristas que se ha merecido un nombre propio: JASP, es decir, jóvenes aunque sobradamente preparados" (CostaPau, 2010c: 3). 
vilístico en 1994, muy similar al creado por Alguacil con la matización de la cantidad salarial, y que se ha incluido a su vez en el indignado de Hessel. "El movimiento 15M [...] agrupa a estudiantes, parados, mileuristas (en el mejor de los casos) y trabajadores sobradamente preparados" (Gil, 2011: web).

En cuanto al término mileurista en sí, resulta oportuno comentar la evolución de su presentación escrita, puesto que nos referimos a un neologismo ${ }^{8}$. De esta manera, si observamos su evolución anual (figura 2), comprobamos como desde la fase de crecimiento hasta la de declive se redacta mayoritariamente en cursiva o itálica, un hecho refleja perfectamente la obsolescencia del término. Sin embargo, en esta última fase que corresponde con el año 2011, al igual que el término llega a normalizarse y aceptarse periodísticamente, en tanto que los tipos redondos superan a las cursivas, el mileurista se generaliza también en la sociedad, como más adelante veremos.

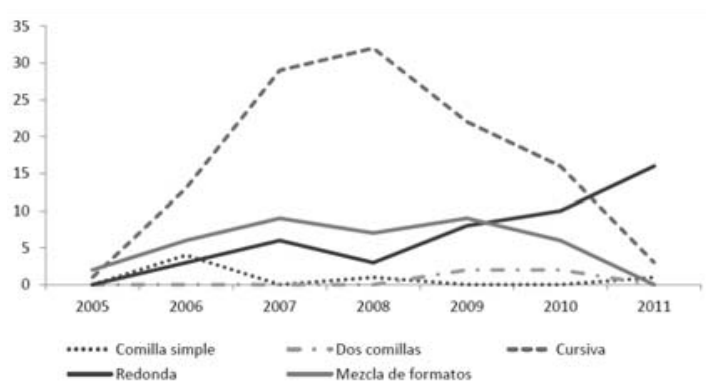

Figura 2. Evolución anual de la presentación escrita del término mileurista. Fuente: Elaboración propia a partir de datos de El País

Por último, queremos comprobar si la definición de mileurista de Alguacil evoluciona o se mantiene en las publicaciones de El País. Su definición recalcaba áreas como la educación, el empleo, la juventud o la vivienda. En cambio, el análisis de las temáticas principales que hemos efectuado refleja un predominio de la temática Política (31\%), pese a que en la definición sea uno de los temas que menos relación guarda con ese concepto. Con cierta entidad le siguen Sociedad (28\%) y Economía (19\%), encontrando, a continuación, una menor presencia en los temas de Vivienda (7\%), Cultura (4\%) y Educación (3\%).

En cambio, el estudio de los subtemas, es decir, aquellas cuestiones más específicas que abarcarían varias de las temáticas principales, ofrece unos resultados que concuerdan mejor con los adjetivos que definen el mileurista de Alguacil: nos referimos a los salarios (14\%) y la juventud (11\%). Entre los subtemas relacionados con ellos encontraríamos las acciones o medidas políticas (10\%), el precio de la vivienda (8\%), el empleo (7\%) y la crisis económica (6\%). En otras palabras, el análisis de los subtemas nos permite analizar y realizar un perfil de la trayectoria del mileurista a lo largo de sus seis años de vida en El País.

${ }^{8}$ El País por medio de su libro de estilo indica que para los neologismos (palabras nuevas de uso no extendido, que deben explicarse) se utilizará la cursiva (El País, 2004: 64). Por el contrario, Barcia (2005: web) considera que convendría entrecomillarlos. 


\section{El perfil del mileurista}

Como ya se ha comentado en reiteradas ocasiones, en agosto del 2005 se publicó por primera vez el término mileurista. El lugar, una carta al director en el diario El País. La autora, una ciudadana hasta entonces desconocida: Carolina Alguacil. En los siguientes apartados procuraremos analizar si aquel perfil descrito por Carolina se ha mantenido, se ha matizado y si ha sido un término adoptado por la sociedad.

\subsection{Ser mileurista $o$ aspirar a serlo}

A lo largo de este tiempo aparecen declaraciones como: "Yo con ser mileurista me conformo" (Álvarez, 2008: 6); "De mayor quiero ser mileurista" (Costa-Pau, 2010a: 1); "Ser mileurista ya no es un oprobio. Se ha convertido en una meta" (Sevillano, 2008: 4); o "Mi hija es mileurista, pero yo lo considero un eufemismo porque nunca ha llegado a cobrar tan redonda cantidad" (Sanchotello, 2006: 16). De esta forma comienza el último testimonio, una carta al director de una madre que pone en entredicho la denominación que se hace al sueldo de su hija. Además, critica que en la declaración de la renta la joven tuvo que devolver 880 euros, "una cantidad que casi se equipara con el sueldo más alto que cobró en el año fiscal correspondiente, 900 euros al mes después de 40 horas semanales". Como éstos, son numerosos los argumentos que nos hablan de una aspiración: llegar a cobrar los 1.000 euros (Crespo, 2006: 14; Doncel, 2007: 68; Gorospe, 2007: 28).

De estos artículos se puede deducir que la representación del mileurista no va siempre asociada al sentimiento de pertenencia, sino que también al de aspiración. Eso sí, en algunos momentos se llegan a manejar conceptos como setecientoseurista (Grau, 2008: 26-27) o dos veces mileuristas (Álvarez, 2007b: 32) para referirse a estas personas.

Sin embargo, en el conjunto del corpus de noticias, empezando por la propia Alguacil, son más numerosas las representaciones de pertenencia: "Soy mileurista"; "yo estoy dispuesto a ser mileurista a perpetuidad", opina Joan Vilaplana i Martí (2009: 34), "siempre y cuando una barra de pan costase 10 céntimos, un café 20 céntimos o un piso de tamaño medio no costase más allá de 30.000 euros".

Un dato interesante se produce al ver la evolución de estas significaciones identitarias: aspirar o pertenecer. Como se comprueba en la figura 3, hasta el año 2010 la consideración de pertenecer al fenómeno del mileurismo supera con creces el número de artículos en los que aparece el sentimiento de meta u objetivo. A partir de 2011 se acercan estas dos posturas: hay muchas menos personas que afirman cobrar entre $900 \mathrm{y}$ 1.100 euros ${ }^{10} \mathrm{y}$, sin embargo, los salarios que no rozan esa cifra mantienen la misma tendencia. Este descenso tan significativo de las personas que se definen como mileuristas

9 "Picnic, el nombre de una editorial mileurista y un estudio de arquitectura con la cuenta corriente en números rojos” (Mañana, 2008: 64); “¿Por qué se le recorta un 5\% a un mileurista funcionario y no a su compañero mileurista temporal?” (Fütterer, 2010: 30).

${ }^{10}$ Un mileurista es toda persona que cobra un salario mensual entre 900 y 1.100 euros (Rubio, 2009: 5). 
se debe a varias razones, todas acentuadas por la crisis económica, entre las que se encuentran la precariedad laboral, los recortes de personal y la congelación de sueldos ${ }^{11}$.

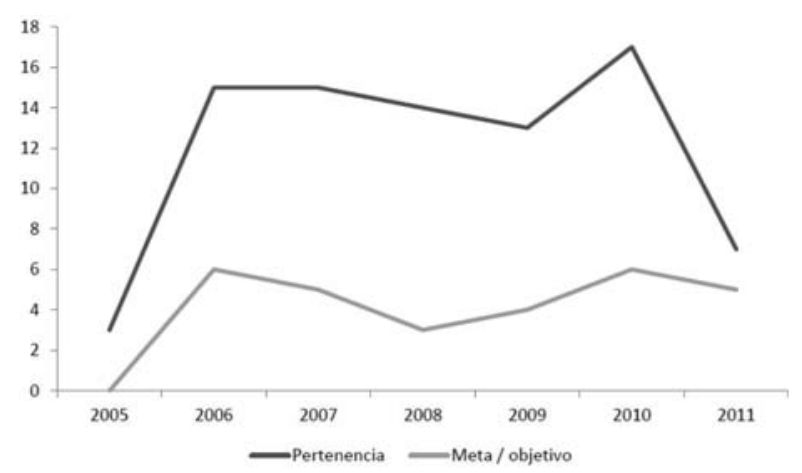

Figura 3. Representaciones del mileurismo (2005-2011). Fuente: Elaboración propia a partir de datos de El País

\subsection{Edad y género}

A continuación analizamos este fenómeno en relación a dos variables sociodemográficas, fundamentales para este perfil, que están muy ligadas con la definición de mileurismo: la edad y el género. La primera de estas variables imprescindibles para analizar en este estudio es la edad. El motivo de ello es que desde la definición publicada por Alguacil hasta las que le pudieron seguir hacen especial hincapié en la edad de los mileuristas. Así, el perfil predominante es el de jóvenes de 25 a 29 años, rasgo que compartía Alguacil cuando acuñó el término mileurista en 2005, y que también concuerda con los datos procedentes del INE. Además, este concepto aparece asociado a todas las franjas de edad, tal y como se aprecia en la figura 4.

\begin{tabular}{|l|c|c|}
\hline \multicolumn{1}{|c|}{ Edad } & $\begin{array}{l}\text { Número de piezas en las que apare- } \\
\text { cen mileuristas (El País. 2005/2011) }\end{array}$ & $\begin{array}{c}\text { Ganancia media anual por trabaja- } \\
\text { dor, en euros (INE - 2009) }\end{array}$ \\
\hline Menos de 20 & 1 & $9.624,47$ \\
\hline $20-24$ & 11 & $12.784,44$ \\
\hline $25-29$ & 27 & $17.530,28$ \\
\hline $30-34$ & 15 & $20.818,91$ \\
\hline
\end{tabular}

${ }^{11}$ Algunos ejemplos: "Hecho: la mitad de los españoles gana menos de 15.760 euros al año, es decir, es mileurista. Hecho: el 30\% de los parados lleva más de un año en esa situación y sólo en el último ejercicio ha crecido en 600.000 personas. Éstos son algunos de los números del principal problema que padece España" (Estefanía, 2009: 6-7); "Un mileurista embargado, por ejemplo, puede estar seguro de que se le va a respetar casi todo su sueldo, mientras que la entidad financiera que quiera cobrar su deuda con alguien que gane 2.500 euros aspirará a detraerle entre 500 y 1.000 euros al mes" (Doncel, 2011: 30); "Me temo que el Estado no me va a considerar mileurista y me aplicará el recorte sobre el total ganado cada mes. Al fin y al cabo, es lo que tienen en cuenta para el IRPF. Qué casualidad, que sólo me tienen por mileurista para el cálculo de mi pensión', ironiza Alexander Perkinks, médico residente de un centro de salud madrileño" (Meyer y García, 2010: 16-17). 


\begin{tabular}{|l|l|l|}
\hline $35-39$ & 4 & $22.824,94$ \\
\hline $40-44$ & 3 & $23.822,65$ \\
\hline $45-49$ & 4 & $24.846,62$ \\
\hline $50-54$ & 1 & $26.182,26$ \\
\hline 55 o más & 6 & $24.763,24$ \\
\hline
\end{tabular}

Figura 4. Análisis de la edad, la frecuencia del mileurismo y el salario.

Fuente: Elaboración propia a partir de datos de El País e Instituto Nacional de Estadística

Como se puede observar, según las noticias en las que se reflejaba la edad de sus protagonistas, la cohorte de edad en la que se registra un mayor número de veces la palabra mileurista es la de 25-29 (27 casos), seguida de la de 30-34 (15) y la de 2024 (11). Si consideramos que la remuneración de un mileurista se mueve entre los 900 y 1.100 euros mensuales o, lo que es lo mismo, unos 15.500 euros anuales, los resultados que se desprenden del estudio de las publicaciones en El País coinciden plenamente con la edad señalada por Alguacil, ya que la tónica general es la que se ha planteado, un perfil de jóvenes treintañeros ${ }^{12}$. Algunos ejemplos extraídos del corpus reflejan muy bien esta situación, como el reportaje de Antonio Jiménez Barca (2005: 1-3) que centra la edad del mileurista en una persona joven que tiene entre 25 y 34 años, pese a que en algunos casos alargue la cifra hasta los 37 años.

Sin embargo, existen otras dos ideas que nos parece interesante destacar en este apartado. Una de ellas es la presencia de mileuristas en todas las cohortes. La segunda, el hecho de que los mayores de 55 también estén presentes en el fenómeno del mileurismo (siendo muchos de ellos jubilados). Este dato es relevante al significar que se ha superado la barrera de la edad para abarcar a toda aquella persona que percibe una remuneración mensual de mil euros.

Por otro lado, en una de las cartas al director, un lector apunta que no le "ha quedado más remedio que seguir cotizando hasta los 65 años... o más, y llegar a los 50 años cotizados para poder recibir una prestación, esta sí, contributiva, que a duras penas llegará a la categoría de mileurista" (Vargas, 2011: 28). Por lo tanto, podríamos concluir este apartado diciendo que el fenómeno del mileurismo ha roto la barrera de la edad. En otras palabras, se ha superado el concepto de generación, pues abarca tanto a los treintañeros como a sus progenitores que se encuentran jubilados en muchos de los casos.

\subsubsection{El género del mileurista}

La segunda variable que nos resulta interesante para analizar en relación al perfil del mileurista es el género del perfil del mileurista. En primer lugar, hay que destacar que

12 Por ejemplo: "Tengo 27 años, una licenciatura, un posgrado y hace seis años que trabajo. Aún no me puedo creer que continúe siendo mileurista" (Pons, 2011: 28); "El malestar de los jóvenes, ese ingrediente que más ha encendido las protestas de los indignados o 15-M, tiene también su colchón estadístico: el único grupo con edad de trabajar en el que hubo una bajada media de los salarios fue entre los empleados menores de 25 años" (Mars, 2011: 22). 
el género, habitual a lo largo del tiempo y por diferentes $\operatorname{motivos}^{13}$, ha sido un factor que ha condicionado la remuneración.

Por este motivo, aunque en las noticias analizadas encontramos más casos de mileuristas hombres (24\%) que mujeres $(21 \%)$, los testimonios publicados por el periódico no dejan lugar a dudas: el mileurismo incide de una manera más elevada en las mujeres que en los hombres. En otras palabras, la evolución a lo largo de estos últimos seis años demuestra que este fenómeno, aunque afecta a ambos sexos, parece tener una mayor incidencia en el género femenino. Así, el mismo periódico publicó una pieza que vinculaba el concepto mileurista a jóvenes, mujeres e inmigrantes (Costa-Pau, 2010b: 3). Se dice en la misma que estos colectivos son los más castigados por la crisis, que constituyen otra forma de pobreza que no tiene en cuenta las titulaciones académicas.

En esta línea, encontramos las siguientes declaraciones que corroboran esta impresión: "Más paro, más contratos temporales y un salario mileurista de media. Así es la vida laboral para las mujeres en Galicia" (Vizioso, 2008: 18), o bien que "el 60\% de los trabajadores andaluces, más de 2.200.000, la mayoría mujeres, no es ni siquiera mileurista" (Pérez Monguio, 2007: 4). Por otro lado, estos datos parecen corroborarse con los publicados por el INE que señalan que el porcentaje de mujeres españolas que cobran un sueldo mileurista $(39,21 \%)$ es superior al de los hombres $(30,04 \%)^{14}$.

\subsection{Salario y situación laboral del mileurista}

Si existe un elemento que marcó aquella primera definición y publicación de Alguacil sobre el mileurismo es el salario, ya que la remuneración es el elemento que constituye el nexo común y a la vez la idiosincrasia de todas esas personas que estamos analizando y perfilando. Así como con la edad, especialmente, y también con el género se podría matizar aquella primera definición de mileurista, no ocurre lo mismo con la remuneración percibida por este grupo. En general, a lo largo de estos seis años analizados, cuando utilizamos la palabra mileurista estamos pensando en una persona que cobra más o menos mil euros: "El salario más común entre los españoles, en cualquier caso, queda

${ }^{13}$ Durán (1982) señala que a la diferencia salarial entre hombres y mujeres contribuyó la creencia en la inferioridad física, intelectual y moral de la mujer, aceptada entre los tratadistas españoles del siglo XV y XVI. Por otro lado, la diferencia salarial presenta una extensa literatura en estudios relacionados con la Revolución Industrial. Por ejemplo, Burnette (1997) afirma que las diferencias salariales suelen llevar a hablar de discriminación salarial. Sin embargó, cree que dicha idea debe matizarse y para ello analiza la diferencia salarial que se produce en las tarifas a tiempo, las tarifas a destajo, y en tercer lugar, estrechamente relacionado con las teorías neoclásicas sobre las diferencias salariales, las relacionadas con la baja productividad y el menor rendimiento de las mujeres, debido a varios factores como son el capital humano, la maternidad y la fuerza. En cambio, otros autores, como Humphries (1991) o Valanze (1995: 89) consideran que existe la opinión de que las mujeres pueden recibir un salario "customary" (acostumbrado) en lugar de un salario de mercado.

${ }^{14}$ En este caso consideramos un salario mileurista en el año 2009 aquel situado entre 1 o 2 veces el Salario Mínimo Interprofesional (SMI). 
en 15.500 euros, el del mileurista" (Mars, 2011: 22). Otro asunto es que, como hemos visto, pertenecer a este colectivo parece ser más una aspiración que una realidad.

En los datos que se pueden extraer de El País, se da un claro predomino de un perfil de mileurista ocupado, que pertenece además al sector servicios ${ }^{15}$. Un dato significativo es el aumento de la frecuencia de aparición de mileuristas del sector servicios a partir de 2008, justo cuando comenzó la crisis. De esta situación se puede concluir que, a raíz de la crisis inmobiliaria, muchos trabajadores de los sectores de la construcción o la industria buscaron trabajo en los servicios ${ }^{16}$. Por tanto, el número de mileuristas también ascendió.

Sin embargo, si analizamos la evolución de los ocupados a lo largo del periodo 2005-2011, como podemos comprobar en la figura 5, apreciamos perfectamente que a medida que pasan los años, la frecuencia de testimonios que se declaran ocupados en el corpus de noticias desciende progresivamente, al mismo tiempo que los mileuristas parados, bien por las pensiones, por cobro del paro o, simplemente, porque lo fueron, tienen un protagonismo mayor.

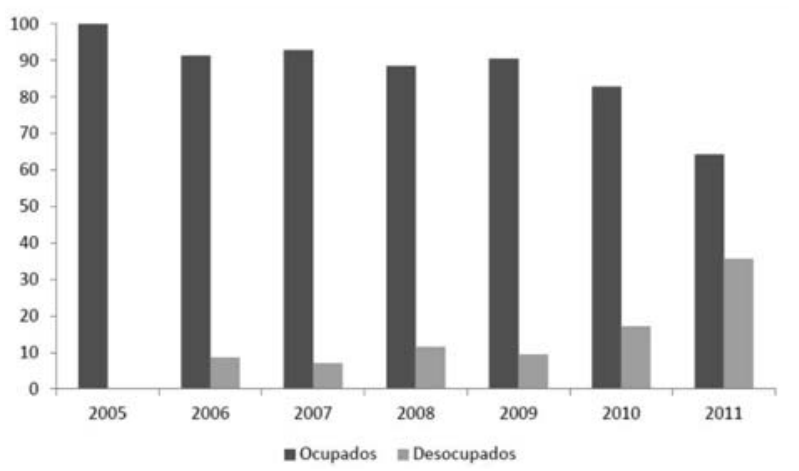

Figura 5. Porcentaje de los mileuristas ocupados y desocupados en El País (2005-2011). Fuente: Elaboración propia a partir de datos de El País

Otra de las características del perfil del mileurista que podemos observar es la precariedad laboral y el abuso sobre el empleado. La mayor parte de las declaraciones se vinculan a las quejas, sobre todo por parte de gente joven, que piden jornadas laborales que les permitan compaginar vida laboral y familiar ${ }^{17}$, contratos permanentes ${ }^{18} \mathrm{y}$

\footnotetext{
${ }^{15}$ Algunos ejemplos del corpus: fisioterapeuta (Banchar, 2006: 33); cantante de jazz (Marimón, 2006: 37); y socióloga (Ayuso, 2006: 31).

16 "La burbuja provocada por el euro financió temporalmente el crecimiento del sector de la construcción, del inmobiliario y de las industrias y servicios dependientes de ambos sectores. Por otra parte, ha dejado incorporados a nuestra economía el crecimiento del empleo de las Administraciones Públicas y el de los sectores que prestan servicios sociales" (Recarte, 2011: web).

${ }^{17}$ Según Amparo Mencía, las empresas "prefieren a un joven mileurista" (Sánchez-Vallejo, 2007: 30-31).

${ }^{18}$ En un sondeo del CIS (2009: web) sobre la juventud, el 64,8\% de los jóvenes encuestados dijo que prefería un contrato permanente a tiempo parcial que uno temporal de jornada completa en el año 2009.
} 
sueldos acordes a su formación académica ${ }^{19}$, ya que "el $40 \%$ de los titulados universitarios no llega ni tan siquiera al estatus de mileurista" (Mellado, 2008: 7). En este sentido, Recio (2007: 425), al analizar la situación laboral de los jóvenes, comenta que "efectivamente hay un aumento de lo que llamamos inseguridad económica y ésta afecta de forma poderosa a los jóvenes que se incorporan al mercado laboral bajo las nuevas reglas de la flexibilidad y la desregulación".

Por lo tanto, la inseguridad económica y la precariedad laboral son una constante en los artículos analizados, ya que en estos factores se ubica el origen de muchos de los problemas o de las decisiones que toman ahora los jóvenes: "sucesión de contratos temporales o becas, salarios que no se acercan al mileurismo, falsos autónomos, pagos en B para esquivar la cotización, contratos que terminan en julio y se renuevan en septiembre, etc. ${ }^{20}$.

\subsection{Formación académica de los mileuristas}

Muy relacionado con la situación económica y laboral de los mileuristas está el nivel de formación. En la definición de Alguacil (2005: 11) podíamos leer que "el mileurista es aquel joven [...] licenciado, bien preparado, que habla idiomas, tiene posgrados, másteres y cursillos". Como en éste, los ejemplos se repiten: "Marian Villa, doctora e investigadora, vive de becas. Trabaja 10 horas al día en el laboratorio por 1.100 euros al mes. 'No lo dejo porque sería tirar ocho años a la basura" (Delgado, 2010: 16) ${ }^{21}$. Así, en los artículos analizados, las personas que son catalogadas como mileuristas, y de las cuales se menciona el tipo de formación, poseen un título universitario en el $89,2 \%$ de los casos. Dichos universitarios son en su mayoría jóvenes entre 25 y 34 años, la población que aparece con más frecuencia en el corpus de los artículos.

Su situación de mileuristas está muy relacionada con la precariedad, como se comentaba anteriormente, especialmente con un aspecto que va parejo a la formación académica: la sobrecualificación: "En pocos países europeos hay tantos licenciados, máster arriba máster abajo, con sueldos mileuristas" (Mars, 2008: 22)22; o cuando leemos "esforzarse en los estudios y cursar una carrera universitaria ha dejado de ser un ascensor social, ni siquiera es la garantía de un buen futuro laboral [...] no se arrepienten del camino andado, pero saben que han de pelear su futuro pese a las calificaciones alcanzadas. Muchos son conscientes de que engrosarán las filas de los mileuristas" (Aunión, 2007: 35). Tanto es así, que en 2007 este fenómeno afectó, según la consultora Accenture, a más de un $60 \%$ de los jóvenes españoles, que no pudieron

19 "La universidad forma mano de obra barata" (Mañana, 2008: 64).

20 "Esteban Sánchez Moreno, sociólogo de la Universidad Complutense de Madrid, afirma que las personas entre 20 y 30 años asumen la precariedad laboral como algo natural. No es que sean acríticos, es que han dado por hecho que es algo inevitable por lo que tienen que pasar" (Delgado, 2010: 16).

${ }^{21}$ En esta línea podemos leer numerosas noticias, como "soy un privilegiado licenciado que gana un sueldo mileurista por un trabajo de 40 horas semanales. Y me estremece saber que hay gente que cobra por una hora lo que yo sufro un mes en ganar" (Díaz, 2010: 26).

${ }^{22}$ En otro caso comprobamos: "Másteres, estudios de posgrado, doctorados, idiomas [...] el signo de identidad de esta generación Peter Pan” (Muñoz, 2009: 4). 
elegir un empleo vinculado a sus estudios. Un $48 \%$ aceptó lo primero y único que se le ofreció (Navarro, 2007: 6-12; Willisch, 2009: 6).

El descontento que los universitarios muestran en los textos de El País se debe precisamente a que esperaban que sus estudios les proporcionaran un mejor nivel de vida que al resto de la población no universitaria: “Tengo 27 años, soy Doctor en Química y mileurista. [...] Me doy cuenta que después de ocho años de educación básica, cuatro de secundaria, cuatro de universitaria y cinco de doctorado me considero un experto en Química Analítica y también en juegos de azar. ¿Realmente era necesario tanto para no lograr mucho? [...] Quizás no fueran necesarios tantos años de estudio" (Mellado, 2008: 7).

Sin embargo, "el mileurismo [...] ha dejado de ser un terreno exclusivo para jóvenes universitarios recién licenciados que tienen que aceptar bajos salarios para hacerse un currículo laboral. En los últimos años ha incorporado a obreros cualificados, parados de larga duración, inmigrantes, empleados, cuarentones expulsados del mercado laboral y hasta prejubilados. Se estima que en España pueden alcanzar en torno a los doce millones de personas" (Muñoz, 2009: 4).

Por lo tanto, a pesar de que los datos extraídos de las noticias de El País reflejan la precariedad laboral casi únicamente en el sector universitario, la realidad nos muestra que el mileurismo ya no es solo una seña de identidad de los licenciados. Eso sí, lo que parece observarse es que en tiempos de crisis los trabajadores con menos formación especializada presentan más flexibilidad a la hora de desempeñar un puesto de trabajo, y con ello tienen la posibilidad de encadenar una sucesión de contratos temporales, con salarios que en muchas ocasiones no se acercan al mileurismo, que les permite superar tiempos difíciles mejor que a los licenciados (OBJOVEM, 2010).

En este sentido, en coyunturas económicas más o menos estables y favorables las condiciones laborales, en referencia a los trabajos indefinidos, se incrementan de un modo paralelo al nivel de estudios. En definitiva, la formación parece ser un seguro de crecimiento profesional y salarial, ya que, como podemos leer en la siguiente noticia, "a los 17 años, un adolescente balear puede ganar lo mismo que un universitario vasco de 26 años mileurista, pero cuando pasen tres años, él seguirá con casi el mismo sueldo y el del vasco será bastante más"23.

No obstante, lo que parece concluyente es que los jóvenes, tengan la formación que tengan, forman uno de los grupos más afectados por la crisis económica. Un estudio prospectivo de la OECD (2011: web) sobre el nivel de paro en España respecto al año 2011 afirma que "las tres cuartas partes de los desempleados a largo plazo, con más de 12 meses de paro, son trabajadores jóvenes, y casi dos tercios de los mismos no tienen experiencia".

\subsection{Vivienda. ¿Con mis padres, solo o compartiendo?}

La vivienda es otra de las características que conforman este perfil tipo del mileurista. ¿Dónde y con quién viven los mileuristas? Entre 2003 y la primera mitad de 2009 se

${ }^{23}$ Así, lo explica Mercedes Esteban, vicepresidenta de la Fundación Europea Sociedad y Educación (Silió, 2008: 27). 
produjo en España un incremento de jóvenes que lograron establecerse en una vivienda independiente, en parte por el ciclo expansivo de la economía, la consiguiente rápida incorporación de los jóvenes al mercado laboral y la llegada de inmigrantes que, a menudo, dejaban a sus familias en sus países de origen y, por consiguiente, estaban emancipados (OBJOVI, 2011).

Sin embargo, a partir de entonces, la tasa de emancipación ha ido retrocediendo: el elevado precio de la vivienda, la crisis económica y las elevadas tasas de paro son los principales factores. Tampoco se puede ignorar la dependencia familiar de los jóvenes españoles, que tienen la posibilidad de vivir en familias centradas en la provisión de su bienestar, y que a la hora de decidir emanciparse buscan un nivel de vida mejor o como mínimo igual que el de su familia de origen, evitando movimientos descendentes en la escala social (Gentile, 2010: 181).

Por ello, la dificultad de acceso a la vivienda ha llegado a representar el tercer problema más importante después del paro y la crisis económica (CIS, 2009: web). Lo leemos en un titular: "Mileurista, busca piso... y no lo encuentra" (Doncel, 2006: 37). Esta preocupación queda recogida en diferentes testimonios publicados en El País. Uno de ellos es el de Anna Rovira, que se define como estudiante, activista, camarera, que no llega a fin de mes y que tiene que compartir piso. O bien el de Isabel Molina (2007: 14) que en una carta al director cuenta su aventura al intentar pasar de compartir piso a vivir sola. Siendo mileurista, solo para cubrir gastos iniciales burocráticos necesita 6.730 euros. Además, luego se suman otros gastos derivados del contrato.

Para ellas, como para muchos otros jóvenes, "el acceso a la vivienda es una de las cuestiones que más le preocupan" (Blanchar y Pellicer, 2006: 32), hasta tal punto que conseguir una vivienda se considera como si a uno le hubiera tocado la lotería $\mathrm{a}^{24} \mathrm{o}$ incluso "mejor" (Álvarez, 2007a: 28). Por lo tanto, entre los mileuristas encontramos dos grandes tipos de situaciones: los que no han conseguido abandonar el hogar paterno y los que a pesar de todas las dificultades sí han dado ese paso.

Así, en los artículos analizados, encontramos que muchos jóvenes se encuentran en la primera de esas posibilidades. Un titular remarca: "Independizarse, un querer y no poder" (Ovelar, 2006: 29). Los resultados determinan que el 25\% de los jóvenes viven en casa de los padres, pues no pueden permitirse un alquiler. En este sentido, Gentile (2010: 181) afirma que "el desarrollo limitado de las políticas sociales y el contexto poco halagüeño, respecto a la seguridad que se disfruta en familia, hacen que la emancipación de los jóvenes españoles sea más complicada y tardía en comparación con sus coetáneos europeos" 25 .

24 “A Isabel Cidonca, de 35 años, mileurista, le tocó la lotería en 2004: una vivienda de protección oficial en el centro de Barcelona" (Pérez, 2007: 88).

${ }^{25}$ Entre los textos analizados encontramos diversos testimonios que denuncian la escasez de políticas sociales, como por ejemplo en la noticia que se podía leer "como en una performance o en un zoo, un joven ejemplar de mileurista fue enjaulado ayer en una infravivienda de 26 metros cuadros por el Consejo de la Juventud, frente al Museo Reina Sofia. Era la manera de protestar por la insuficiente política de vivienda de la Comunidad de Madrid" (García, 2007: 32). Además, "las escasas ayudas sociales a la familia, como el cheque bebé, no facilitan el tener hijos" (Pérez, 2008: 18). 
A pesar de las dificultades, el tipo de hábitat predominante entre los mileuristas de los artículos de El País hace referencia a las parejas, mayoritariamente jóvenes, que han conseguido emanciparse de sus padres, un $41 \%$ del total, ya tengan hijos, o no, al considerar que no poseen la garantía laboral ni el suficiente presupuesto económico para mantenerlos ${ }^{26}$. Otro tipo de mileurista es aquel representado por otros muchos jóvenes que se han acostumbrado a un nivel de vida sencillo y que tiene como objetivo cubrir primordialmente las necesidades básicas ${ }^{27}$. Son todos aquellos que protagonizan el $20 \%$ de los textos de El Pais: las personas que comparten piso. Es el caso de Elena, que vive con cuatro personas y paga 300 euros al mes por su habitación (Delgado, 2010: 16), y de otros tantos jóvenes, sean estudiantes o no, a los que solo les llega para alquilar habitaciones ${ }^{28}$.

Por último, en el porcentaje más pequeño (un 14\%) se encuentran todos los jóvenes que se han emancipado de sus padres y viven solos ${ }^{29}$. Este dato no es de extrañar si tenemos en cuenta el salario medio de un mileurista, 15.500 euros anuales, ya que según datos del INE (2010: web), al hacer balance del tipo de hogar y el gasto medio por persona, "el mayor valor se registra en hogares unipersonales, con 21.596 euros, cuando la persona es menor de 65 años, y 15.182 euros cuando tiene 65 años o más".

Por todo ello, no es de extrañar que desde que se bautizó a este grupo, entre sus reivindicaciones se halle la demanda de una vivienda: "Como en una performance o en un zoo. Un joven ejemplar de mileurista fue enjaulado ayer en una infravivienda de 26 metros cuadros por el Consejo de la Juventud, frente al Museo Reina Sofía. Era la manera de protestar por la insuficiente política de vivienda de la Comunidad de Madrid" (García, 2007: 32).

\subsection{Mileurista y clase social: "Adiós, clase media, adiós"30}

Por otro lado, el mileurismo parece haber roto, además de la barrera de "generación", la línea del concepto de clase. Un concepto nacido de las revoluciones burguesas y li-

${ }^{26}$ Entre las dificultades existentes para los jóvenes que se han independizado está el pago de la vivienda (Pérez, 2006: 109).

${ }^{27}$ Por medio de una carta al director, Natacha Manent (2010: 28) resume así la situación de una población dependiente, acostumbrada a un nivel de vida y a un consumo que, en su opinión, es la causa de la crisis: "Soy una trabajadora normal y tengo unas necesidades que, a mi juicio, considero básicas. No renuevo mi vestuario cada temporada, no tengo tele de plasma y utilizo el mismo móvil desde hace siete años. [...] Mi objetivo es poder ser mileurista y despojarme de necesidades superfluas para poder trabajar menos y disfrutar de la vida. Y no me quejo".

28 "El alquiler medio en Barcelona cuesta 915 euros. Estos precios plantean un pequeño problema: el $70 \%$ de los jóvenes catalanes son inframileuristas, según datos sindicales. Así que pagar 900 euros mensuales está a años luz de sus posibilidades" (Delgado, 2010: 1).

29 "Las viviendas también se destinan a becarios e investigadores como Carmen Pérez, que tiene 29 años y es profesora ayudante en el departamento de Trabajo Social. Ella es mileurista. Ahora paga 400 euros al mesa para poder vivir sola en un piso de alquiler en Alicante" (Molto, 2008: 11). En una carta al director leemos: "Alquilar también es un lujo" (Molina, 2007: 14).

${ }^{30}$ Para este epígrafe hemos tomado el titular del reportaje elaborado por Muñoz (2009: 4). 
berales hace unos 200 años. Uno de los aspectos que diferenciaba aquellas nuevas clases sociales de los grupos estamentales del Antiguo Régimen era que frente al mérito de haber nacido en uno u otro estamento ahora se había establecido uno nuevo: la riqueza, la cual no dependía del origen, sino del trabajo de la persona. Por lo tanto, el nacimiento ya no era un obstáculo para el desarrollo profesional o social y, por ello, la posibilidad de crear una sociedad más homogénea, desde el punto de vista de la riqueza, fue posible.

Sin embargo, en la actualidad, tanto en la sociedad española, como en la europea occidental, que parecían haber logrado crear una sociedad en la que predominaba la clase media, la situación parece haber retrocedido, ya que "la desigualad en España se sitúa por encima de la media de la OCDE" (Mars, 2011: 19), especialmente por la diferencia salarial ${ }^{31}$ que parece volver a instaurar el inmovilismo social (Iori y Berástegui, 2010: 24-25). "Socialmente, el mileurismo significa el fin de la clase media. Hijos de personas que se han criado en el difuso espectro de la medianía se encuentran frente a una sociedad en la que la dispersión y la especialización profesional han creado compartimentos estancos en los que resulta difícil prosperar" (Soto, 2008: 32).

La desesperación de los afectados se recoge en diferentes testimonios. Así, Rita, una mileurista de 34 años que trabaja en un supermercado en un barrio del este de Berlín, manifestaba que hacía años que tenía el mismo sueldo: “¿Para qué? ¿Para que los millonarios se lleven el dinero a sus bancos en el extranjero?" (Güell, 2008: 36). La situación para los mileuristas no es fácil, especialmente para los jóvenes, ni a corto ni a largo plazo, por un lado, como hemos visto por la precariedad laboral, y, por otro, cuando se preguntan qué sucederá dentro de 20 o 30 años, cuando se jubilen, siendo que en la actualidad no tienen un empleo estable. En otras palabras, en la actualidad nos encontramos con la paradoja de estar ante una generación de jóvenes que está mejor preparada que nunca y cuyo futuro es el más incierto y con menos perspectivas de superar el nivel de vida de la generación de sus padres.

A pesar de este presente y futuro incierto, donde cada vez más las diferencias sociales se acentúan y la clase media, que hasta ahora había sido mayoritaria, parece ir difuminándose, hay que apostar por los jóvenes mileuristas: "La nueva clase media que la sustituya [a esa misma clase media] bien pudieran ser los pujantes mileuristas, los que ganan mil euros al mes. Tal y como sucedió cuando los mamíferos sustituyeron a sus gigantes antecesores, los mileuristas tiene una mayor capacidad de adaptación a circunstancias difíciles" (Muñoz, 2009: 4). A pesar de ello, "que en este país se considere privilegiado a quien tiene un sueldo mileurista debería darle alguna pista de por dónde se mueve la sociedad" (García, 2011: 26).

${ }^{31}$ Por medio de una carta al director, un lector señala un amplio abanico de profesiones y apunta que "hoy en día, probablemente, ninguno de ellos llegará a mileurista, muchos ni lo soñarán” (Rodríguez, 2007: 16). Además, “entre el año 2000 y el año 2008, época en la que se acuñó el término mileurista, aumentó un 32\% el número de asalariados y descendió un 2,1\% el de empresarios" (Mandía, 2010: 2). 


\section{Conclusión}

El estudio de la evolución, tratamiento y representación del término mileurista en $E l$ País durante el periodo comprendido entre el 21 de agosto de 2005, momento en el que fue publicado por primera vez, y el 20 de noviembre de 2011, coincidiendo con las elecciones generales, nos ha permitido establecer una serie de paradigmas que definen a las personas denominadas mileuristas dependiendo de su remuneración monetaria, edad, formación, hábitat o situación laboral.

Sin embargo, el análisis del tratamiento de este neologismo nos ha permitido evidenciar que el ciclo de la vida de dicho término no ha emanado de la crisis económica en la que estamos actualmente insertos, sino que surgió en una todavía aparentemente bonanza y un crecimiento de la economía española de los más altos de Europa. A pesar de ello, esta coyuntura desfavorable trajo consigo un cambio, que no solo afectó a su ciclo de vida, sino también a su lado distintivo, a quién representa, y a su runa, pues pasó de tipos cursivos o entrecomillados a redondos.

Lo que comenzó siendo una definición que abarcaba especialmente a jóvenes treintañeros, aparentemente formados y con salarios medios mensuales de unos mil euros ha evolucionado en dos direcciones. Una de ellas, es que el término ha llegado a englobar todas las posibles franjas de la población activa, incluso de los retirados (mayores de 65 años), y a los profesionales universitarios y no universitarios. Además, en este escenario se comprueba que la especialización, primordialmente la universitaria, es menos flexible en un mundo laboral cada vez más encorsetado, por lo que a corto plazo dificulta las opciones laborales. En ambos casos, siguiendo las teorías de la desigualdad salarial por género que apuntan diferentes estudios, la mujer se ve más afectada que el hombre en esta nueva situación. La realidad que proporcionan los textos analizados evidencia que al hablar entre sexos se observa un mayor número de casos que corresponden con hombres.

La segunda y fundamental evolución evidencia que ser mileurista ya no es sinónimo de precariedad por el tiempo y esfuerzo empleados, además de los resultados obtenidos. A diferencia de lo que ocurría en un principio, durante los últimos años ha llegado a convertirse en un estatus, es decir, en la aspiración de gran parte de la sociedad española. Por lo tanto, la tan anhelada y trabajada formación de una clase media en pro de un equilibrio de la riqueza parece ir difuminándose hacia una sociedad en la que las desigualdades se acentúan.

Las repercusiones de estos cambios de actitudes y comportamientos no sólo incurren en el mercado laboral, también afectan e inciden en otras esferas, como las sociodemográficas ya que, entre otras, la edad a la que nos independizamos tiende a incrementarse, a retrasarse la maternidad-paternidad, y a reducirse la natalidad. Si tenemos en cuenta que España es hoy uno de los países más envejecidos del mundo desarrollado, este hecho no vendrá más que a agravar la crónica enfermedad que padecemos y que parece queremos obviar constantemente.

\section{Referencias bibliográficas}

ALGUACIL, Carolina (2005): "Yo soy 'mileurista", en El País, 21 de agosto, Carta al Director, p. 11. 
ÁLVAREZ, Pilar (2007a): “Ana y Pedro ya tienen casa”, en El País, 9 de abril, p. 28.

ÁLVAREZ, Pilar (2007b): "Los madrileños, dos veces 'mileuristas”, en El País, Suplemento Madrid, 30 de septiembre, p. 32.

ÁLVAREZ, Pilar (2008): “Albañil municipal de reserva... y por sorteo", en El País, Suplemento Madrid, 4 de enero, p. 6.

ASOCIACIÓN DE EDITORES DE DIARIOS ESPAÑOLES (2010): Libro Blanco de la Prensa Diaria 2010. Madrid: AEDE.

AUNIÓN, Juan Antonio (2007): “¿Para qué sirven los sobresalientes?”, en El País, 26 de agosto, p. 35.

AUNIÓN, Juan Antonio (2011): “Indignados, pero aún poco movilizados" en El País, 17 de abril, p. 26-27.

AYUSO, Gloria (2006): “Mileurista/socióloga”, en El País, 31 de octubre, p. 31.

BANCHAR, Clara (2006): "Mileurista/fisioterapeuta", en El País, 18 de octubre, p. 33.

BARCIA, Pedro Luis (2005): "Tratamiento de neologismos y extranjerismos en el DPD", en Archivo de noticias académicas de la Real Academia Española: http://www.rae.es/rae/gestores/gespub000009.nsf/\%28voAnexos\%29/archC4B2B4 3F4803FF78C125715D003899B8/\$FILE/PRESLUISBARCIA.HTM [fecha de consulta: 3 de mayo de 2012].

BERELSON, Bernard (1952): Content Analysis in Communication Research. Nueva York: Free Press.

BURNETTE, Joyce (1997): "An investigation of the female-male wage gap during the Industrial Revolution in Britain", en Economic History Review, vol. L, nº. 2, pp. 257-281.

CÁCERES ZAPATERO, María Dolores (2011): "La construcción social de la realidad: el papel de los profesionales en el establecimiento de la agenda temática", en Estudios sobre el mensaje periodístico, vol. 17, $\mathrm{n}^{\circ} .2$. Madrid, Servicio de Publicaciones de la Universidad Complutense, pp. 303-324.

CASALS CARRO, María Jesús (2004): "La opinión en la prensa: retrato de España en el primer año del siglo XXI", en Estudios sobre el mensaje periodístico, vol. 10. Madrid, Servicio de Publicaciones de la Universidad Complutense, pp. 9-66.

CENTRO DE INVESTIGACIONES SOCIOLÓGICAS (2009): "Sondeo sobre la juventud, 2009 (II)", en CIS, estudio $\mathrm{n}^{\circ}$ 2.822, noviembre de 2009: http://www.cis.es/cis/opencms/_Archivos/Marginales/2820_2839/2822/ft2822.pdf [fecha de consulta: 3 de mayo de 2012].

CÓRDOVA JIMÉNEZ, Alejandro (2011): "Las cartas al director como género periodístico", Revista ZER, vol. XVI, nº 30, p. 189-202.

COSTA-PAU, Marta (2010a): "El sueño de ser 'mileurista”, en El País, 7 de febrero, Suplemento Cataluña, p. 1. 
COSTA-PAU, Marta (2010b): 'El 'inframileurismo' se ceba en inmigrantes, jóvenes y mujeres", en El País, 7 de febrero, Suplemento Cataluña, p. 3.

COSTA-PAU, Marta (2010c): "La lucha por llegar a fin de mes", en El País, 7 de febrero, Suplemento Cataluña, p. 3.

CRESPO, Esther (2006): "Sobre las VPO en Toledo", en El Pais, Carta al Director, 15 de noviembre, p. 14.

CYTRYNBLUM, Alicia (2009): Periodismo social. Una nueva disciplina. Buenos Aires, La Crujía.

DELGADO, Cristina (2010a): "Paciencia de 'mileurista' por una casa", en El País, 5 de abril, Suplemento Cataluña, p. 1.

DELGADO, Cristina (2010b): "Precariedad, fuente de la eterna juventud", en El País, 20 de septiembre, p. 16.

DÍAZ, Daniel (2010): "El salario de los controladores", en El País, 23 de enero, Carta al Director, p. 26.

DONCEL, Luis (2006) "Mileurista, busca piso... y no lo encuentra, en El País, 1 de octubre, Suplemento Madrid, p. 37.

DONCEL, Luis (2007): “Atrapados por el Euríbor”, en El País, 2 de septiembre, p. 68.

DONCEL, Luis (2011): "Medidas que alivian, pero no curan", El País, 3 de julio, p. 30.

DURÁN, María Ángeles (1982): "El papel de la mujer en la estructura demográfica y económica en el Antiguo Régimen has el Renacimiento", en Análisis e Investigaciones Culturales, Madrid, $\mathrm{n}^{\circ} 11,1982$, pp. 11-20.

EL PAÍS (2004): Libro de estilo de El País. Madrid: Ediciones El País.

ESTEFANÍA, Joaquín (2009): “LLa normalidad?: recuperación sin empleo”, en El País Domingo, 27 de diciembre, pp. 6-7.

FREIRE, Espido (2006): Mileuristas. Retrato de la generación de los mil euros. Barcelona, Ariel.

FÜTTERER, Agnes (2010): “Lo más fácil, pero no lo más justo", en El País, 12 de julio, Carta al Director, p. 30.

GALTUNG, J. y RUGE, M. H. (1980): "La struttura delle notizie dall'estero", en BALDE, P. (ed.), "Il giornalismo como profesione", Milán: Il Saggiatore.

GARCÍA, Íñigo (2007): "Mileurista en el zoo", en El País, 14 de septiembre, Suplemento Madrid, p. 32.

GARCÍA, José María (2011): “Otra vez moderación salarial”, en El País, 14 de agosto, Carta al Director, p. 26.

GARRIGA, Josep (2009): “La generación 'peter pan' está hipotecada”, en El País, 25 de octubre, p. 32. 
GENTILE, Alessandro (2010): "De vuelta al nido en tiempos de crisis. Los boomerang kids españoles", en Revista de estudios de juventud, vol. 90, pp. 181-203.

GIL, Joaquín (2011): "Las caras de la indignación", El País, 17 de mayo, Versión web: http://politica.elpais.com/politica/2011/05/17/actualidad/1305656044 _210451.html [fecha de consulta: 3 de mayo de 2012].

GOROSPE, Pedro (2007): "Llevo 33 años trabajando y no llego a mil euros", en $E l$ País, 2 de mayo, Suplemento País Vasco, p. 25.

GRAU, Abel (2008): "La chispa griega o un nuevo mayo del 68", en El País, 22 de diciembre, pp. 26-27.

GÜELL, Oriol (2008): "El DVD que acorraló a mil millonarios", en El Pais, 24 de febrero, p. 36.

HOLDING, Peter y ELLIOTT, Philip (1979): Making the News. Londres, Longman.

HUMPHRIES, Jane (1991): "Lurking in the wings...: women in the historiography of the Industrial Revolution", en Business \& Economic History, n 20, pp. 32-44.

INSTITUTO NACIONAL DE ESTADÍSTICA (2010): España en cifras 2010. Madrid, Instituto Nacional de Estadística.

IORI, Ricardo y BERÁSTEGUI, Jorge (2010): “Ascensor social: fuera de servicio”, en El País, 11 de agosto, pp. 24-25.

JIMÉNEZ BARCA, Antonio (2005): "La generación de los mil euros", en El País Domingo, 23 de octubre, pp. 1-3.

KRIPPENDORFF, Klaus (1990): Metodología de análisis de contenido. Teoría y práctica. Barcelona, Paidós.

LANG, Gladys y LANG, Kurt (1981): "Watergate. An exploration of the AgendaBuilding Process", en WILHOIT, G. C. y DEBOCK, H. (eds.): Mass Communication Review Yearbook 2. Beverly Hills, CA, Sage.

LÓPEZ-ESCOBAR, Esteban y SÁNCHEZ DE LA YNCERA, Ignacio (1990): “Contexto y selección informativa”, en Comunicación y Sociedad, vol. III, pp. 135- 169.

MANDIÁ, Diana (2010): "El 1\% de los gallegos acapara el 30\% del PIB de las rentas empresariales y de capital", en El País, 11 de noviembre, Suplemento Galicia, p. 2.

MANENT, Natacha (2010): "No estamos en crisis, somos la crisis", en El País, 10 de octubre, Carta al Director, p. 28.

MAÑANA, Carmen (2008): "La editorial mileurista”, en El País, 1 de febrero, p. 64.

MARIMON, Silvia (2006): "Mileurista/cantante de jazz", en El País, 22 de octubre, p. 37.

MARS, Amanda (2008): “Mileuristas' para siempre”, en El País, 11 de diciembre, p. 28.

MARS, Amanda (2011): "La brecha salarial entre directivos y empleados creció en lo peor de la crisis", en El País, 23 de junio, p. 22. 
McCOMBS, Maxwell y SHAW, Donald (1972): "The Agenda-Setting Function of the Mass Media”, en Public Opinion Quarterly, n 36, pp. 176-187.

MELLADO, Sergio (2008): "Una vía rápida al mileurismo", en El País, 17 de junio, Suplemento Andalucía, p. 7.

MEYER, Luis y GARCÍA, Pablo (2010): "No sé si el Gobierno me va a quitar 50 euros al mes o 300", en El País, 16 de mayo, pp. 16-17.

MOLINA, Isabel (2007): “Alquilar también es un lujo" en El País, 12 de enero, Carta al Director, p. 14.

MOLTO, Ezequiel (2008): “Llegan los pisos universitarios", en El País, 7 de marzo, Suplemento Comunidad Valenciana, p. 11.

MOTIS, Miguel Ángel; FERNÁNDEZ, Cayetano; PRIETO, Antonio y CORRAL, Alfonso (2010): "Paradigmas y estereotipos de los inmigrantes en Aragón a través de la prensa (2001-2010)", en Actas del II Congreso Internacional Latina de Comunicación Social, Tenerife, Universidad La Laguna.

MUÑOZ, Ramón (2009): “Adiós, clase media, adiós”, en El País, 31 de mayo, p. 4.

NAVARRO, David (2007): "El paro juvenil”, en El escaparate. Escritura pública, no 48, pp. 6-12.

OBSERVATORIO JOVEN DE EMPLEO EN ESPAÑA (2010): Condiciones de empleo y de trabajo de la juventud en España. $4^{\circ}$ trimestre de 2010. Madrid, Consejo de la Juventud de España.

OBSERVATORIO JOVEN DE VIVIENDA EN ESPAÑA (2011): Estudio sobre la emancipación de los jóvenes españoles. $2^{\circ}$ trimestre de 2011. Madrid, Consejo de la Juventud de España.

ORGANISATION FOR ECONOMIC COOPERATION AND DEVELOPMENT (2011): “Employment Outlook 2011- How does Spain compare?", en Organisation for Economic Cooperation and Development: http://www.oecd.org/ dataoecd/8/45/48682976.pdf. [fecha de consulta: 3 de mayo de 2012].

OVELAR, María (2006): "Muchos jóvenes madrileños no tienen casa", en El País, 3 de agosto, Suplemento Madrid, p. 29.

PÉREZ MONGUIO, Fernando (2007): "UGT sólo firmará convenios con un salario mínimo mileurista", en El País, 23 de noviembre, Suplemento Andalucía, p. 4.

PÉREZ, Claudí (2006): "La hipoteca sube casi 1.000 euros al año", en El País, 9 de junio, p. 109.

PÉREZ, Claudí (2007): "Madrid, Navarra y El País Vasco encabezan la construcción de viviendas sociales", en El Pais, 5 de febrero, p. 88.

PÉREZ, Claudí (2008): "El cheque bebé me viene bien, pero es electoralista”, en $E l$ Pais, 23 de febrero, p. 18.

PONS, Sonia (2011): "Jóvenes a Alemania", en El País, 13 de febrero, Carta al Director, p. 28. 
RAMONET, Ignacio (2011): La explosión del periodismo. De los medios de masas a la masa de los medios. Madrid, Clave intelectual.

RECARTE, Alberto (2011): "Pasado y presente del desempleo en España", en Libre mercado: http://www.libremercado.com/2011-05-09/alberto-recarte-pasado-y-presente-del-desempleo-en-espana-59631/ [fecha de consulta: 3 de mayo de 2012].

RECIO, Albert (2007), “La situación laboral de los jóvenes", en Architecture, City and Environment (ACE), vol. II, $\mathrm{n}^{\mathrm{o}} 5$, pp. 411-426.

REVILLA CASTRO, Juan Carlos (2001): "La construcción discursiva de la juventud: lo general y lo particular". Papers, 63/64, pp. 103-122.

RODRIGO ALSINA, Miquel (1989): La construcción de la noticia, Barcelona, Paidós.

RODRÍGUEZ, Rafael (2007): "Una clase media empobrecida", en El País, 20 de junio, Carta al Director, p. 16.

RUBIO ARRIBAS, Javier (2009): “Aspectos sociológicos del desempleo y la precariedad laboral", en Nómadas. Revista Crítica de Ciencias Sociales y Jurídicas, $\mathrm{n}^{\circ}$ 24, pp. 55-64.

SÁDABA, Teresa (2007): Framing: el encuadre de las noticias. El binomio terrorismo-medios. Buenos Aires, La Crujía.

SÁNCHEZ-VALLEJO, M. Antonia (2007): "Las ejecutivas tiran la toalla. El 30\% de las directivas renuncian a su cargo al no poder conciliar trabajo y familia", en $E l$ País, 10 de noviembre, pp. 30-31.

SANCHOTELlO, Amparo (2006): "Hacienda y los mileuristas", en El País, 16 de junio, Carta al Director, p. 16.

SANTESMASES MESTRE, Miguel (2007): Marketing: conceptos y estrategias, Madrid, Pirámide.

SEVILLANO, Elena (2008): "La mitad de los asalariados gana menos de 1.000 euros", en El País, 12 de febrero, Suplemento Madrid, p. 4.

SIERRA CABALLERO, Francisco (1999): Elementos de la Teoría de la Información. Sevilla, Mad.

SILIÓ, Elisa (2008): "El abandono escolar ya pasa factura", en El País, 04 de octubre, p. 27.

SOTO IVARS, Juan (2008): “Mileuristas' y 'mediocristas”, en El País, 29 de mayo, Carta al Director, p. 32.

VALANZE, Deborah (1995): The first industrial woman. Oxford, Oxford University Press.

VARGAS MORENO, Julio (2011): "Sobre la justicia de las pensiones", en El País, 12 de enero, Carta al Director, p. 28.

VILAPLANA I MARTI, Joan (2009): "Bajada de sueldo", en El País, 26 de junio, Carta al Director, p. 34. 
VIZOSO, Sonia (2008): "La Xunta subvencionará contratos a mujeres en el metal y la construcción", en El País, 19 de febrero, Suplemento Galicia, p. 18.

WILLISCH, Loli (2009): "Titulados universitarios cobran 700 euros en gimnasios públicos", en El País, 22 de diciembre, Suplemento Galicia, p. 6. 